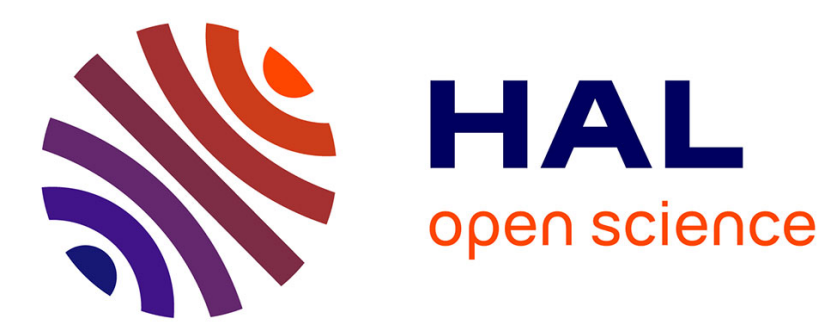

\title{
ON THE EBIC BRIGHT CONTRAST AT THE DOT-AND-HALO FEATURES IN GaAs
}

\author{
C. Frigeri
}

\section{To cite this version:}

C. Frigeri. ON THE EBIC BRIGHT CONTRAST AT THE DOT-AND-HALO FEATURES IN GaAs. Journal de Physique IV Proceedings, 1991, 01 (C6), pp.C6-205-C6-210. 10.1051/jp4:1991631 . jpa00250717

\section{HAL Id: jpa-00250717 https://hal.science/jpa-00250717}

Submitted on 1 Jan 1991

HAL is a multi-disciplinary open access archive for the deposit and dissemination of scientific research documents, whether they are published or not. The documents may come from teaching and research institutions in France or abroad, or from public or private research centers.
L'archive ouverte pluridisciplinaire HAL, est destinée au dépôt et à la diffusion de documents scientifiques de niveau recherche, publiés ou non, émanant des établissements d'enseignement et de recherche français ou étrangers, des laboratoires publics ou privés. 


\title{
ON THE EBIC BRIGHT CONTRAST AT THE DOT-AND-HALO FEATURES IN GaAs
}

\author{
C. FRIGERI \\ CNR-MASPEC Institute, Via Chiavari 18/A, I-43100 Parma, Italy
}

\begin{abstract}
The dot-and-halo features detected by EBIC in Si-doped bulk GaAs have been studied quantitatively by energy-dependent EBIC to determine the influence of the width of the space charge region of the EBIC Schottky diode on the formation of the negative (bright) EBIC contrast. It has been found that in the majority of the cases the bright contrast is mostly due to an increase of the width of the space charge region (i.e. depletion of shallow donor impurities) rather than to an increase of the diffusion length (i.e. depletion of deep non-radiative impurities).
\end{abstract}

\section{1.- Introduction.}

The dot-and-halo contrast at defects is a typical feature detected by EBIC or cathodoluminescence $(\mathrm{CL})$ in semiconductors. As regards EBIC, the bright halos around dislocations have always been ascribed to a depletion of non-radiative deep impurities as the EBIC models so far proposed allowed only the determination of the minority carrier diffusion length $L .1$ The EBIC signal, however, is strongly affected not only by $L$ but also by the width of the space charge region (SCR) associated with the EBIC Schottky diode. ${ }^{2}$ The influence of the width of the SCR, along with some other factors, on the formation of the EBIC bright contrast has been qualitatively discussed in silicon.3,4 On the other hand, for high doping levels (> 1.1018 $\mathrm{cm}^{-3}$ in GaAs) the CL bright halos could often be attributed to a decrease of the dopant density because the CL peak intensity and band-shape parameters (i.e. the slope of the low energy tail of the CL emission band, the peak energy and the half width of the $C L$ band) vary with the electron concentration, 5 although a decrease of the density of the non-radiative centres was also suggested by several authors. 5

The question thus raised is whether and how far the shallow impurities, i. e. the SCR width, can also be involved in the formation of the EBIC bright halos. This can be quantitatively assessed by energy-dependent EBIC efficiency measurements by the method given in Ref. [2] which allows the simultaneous determination of both the diffusion length and the dopant concentration with micron-size spatial resolution.

\section{Experiment.}

The EBIC observations and measurements were carried out in n-type GaAs crystals grown by the Czochralski method from either As- or Ga-rich melts. The samples were silicon doped in the range $1-40.10^{16} \mathrm{~cm}^{-3}$. In order to determine simultaneously and in the same place both the diffusion length and the dopant density, the energy-dependent EBIC method given in 
Ref. [2] has been used. The method is based on the fact that the EBIC current, $I_{C C}$, is the sum of the drift current, $I_{D}$, in the space charge region and the diffusion current, $I_{B}$, in the neutral part of the specimen and is a function of L, SCR width $W$, beam energy $E$ and Schottky metal thickness d

$$
I_{C C}=I_{D}+I_{B}=I_{C C}(L, W, E, d)
$$

so that both $L$ and $W$ can be determined. 2 As the EBIC efficiency measurements are performed by changing the beam energy at the same position, the spatial resolution is of the order of some micrometers, i. e., suitable to study the dot-and-halo features in GaAs. Fig. 1 illustrates the geometry of the method, details of which can be found elsewhere. ${ }^{2}$

The EBIC diodes were prepared by evaporating Au onto the chemically etched sample surfaces. Au-Ge ohmic contacts with resistance $\leq 5 \Omega$ were used. Only those diodes having an ideality factor $\leq 1.05$ were investigated. Such a low ideality factor reasonably guarantees that the fundamental assumption of the energy-dependent EBIC method, i. e., that no carrier recombination occurs within the SCR,2,6 is fulfilled. Beam currents $\leq 0.1 \mathrm{nA}$ were used.

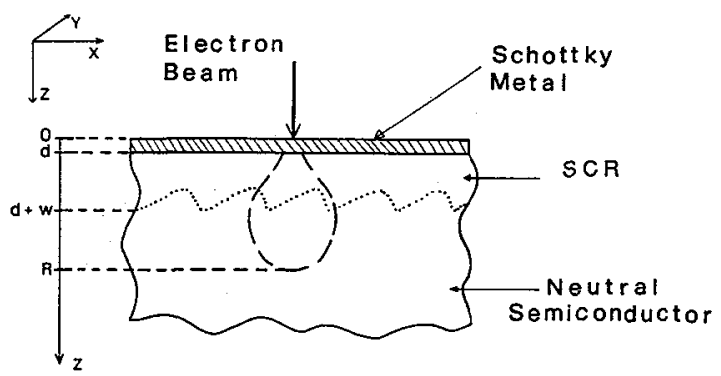

Fig. 1 - Schematics of the geometry of the method

\section{3. - Results and discussion.}

Fig. 2 shows standard dot-and-halo features, i. e., dislocation-related dark dots surrounded by bright halos, in a GaAs crystal grown from an As-rich melt and having an average free electron density $n=2.0 \cdot 10^{17} \mathrm{~cm}^{-3}$. Typical value at $30 \mathrm{keV}$ of the defect contrast, $\mathrm{c}$, defined as $\mathrm{C}=1-\left(\mathrm{I}_{d} / \mathrm{I}_{0}\right), \mathrm{I}_{0}$ being the background EBIC signal and $\mathrm{I}_{d}$ the EBIC signal at the defect, is $+40 \%$ in the dark dot and $-10 \%$ in the bright halo.

Table I gives the typical results of the quantitative EBIC measurements in the dot-andhalo feature $D$ in Fig. 2 . L increases (by only $3 \%$ ) in the bright halo and, as expected, decreases in the dark dot with respect to the surrounding homogeneous semiconductor matrix which is taken as giving the reference values for $L$ and $W$. Table $I$ also shows that the SCR width $W$ increases (by $49 \%$ ) in the bright halo and decreases in the dark dot. The increase of the SCR width W was detected in every bright halo investigated in our samples, irrespective of the doping level. On the other hand, in several bright halos only the SCR width increased significantly whereas $L$ changed by less than $5 \%$, or even remained constant, with respect to the matrix.

These results clearly indicate that the negative (bright) EBIC contrast is due not only to the increase of $L$, as always reported in the literature, but also to the increase of the width of the SCR associated with the diode. The increase of $W$ causes, in fact, the increase of the EBIC signal through the increase of the drift term $I_{D}$ in eq. (1) which is given by 2

$$
I_{D}=\int_{d}^{d+W} F(z, E) d z
$$


where $F(z, E)$ is the carrier generation rate $\left(\mathrm{cm}^{-1} \mathrm{sec}^{-1}\right)$. An increase of the upper limit of integration $d+W$ in eq. (2) is really effective in increasing $I_{C c}$ since all the carriers generated within the SCR are drifted to the SCR edges by the strong electric field therein and are all collected. This is also experimentally true provided the ideality factor of the EBIC diode is as close as possible to 1. A full expression for $\mathrm{I}_{C C}$ has been obtained elsewhere by integration of eq. (2) with the appropriate $F(z, E) .6,7$

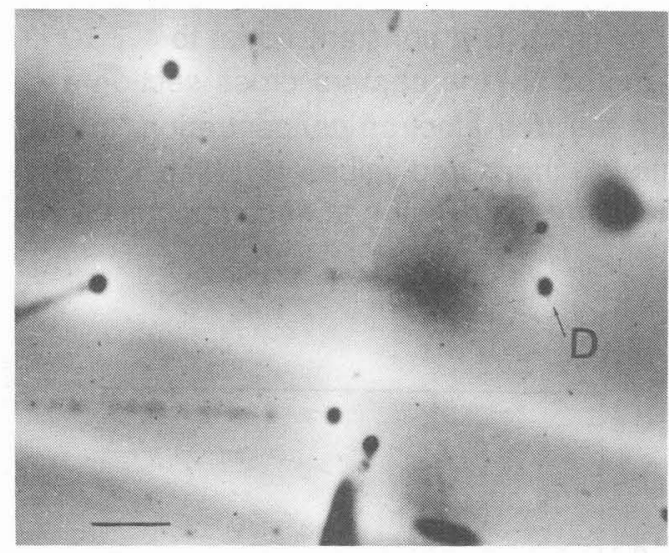

TABLE I

\begin{tabular}{|lccc|}
\hline & $\begin{array}{l}\mathrm{L} \\
(\mu \mathrm{m})\end{array}$ & $\begin{array}{c}\mathrm{W} \\
(\mu \mathrm{m})\end{array}$ & $\begin{array}{l}\mathrm{N}_{\mathrm{D}}-\mathrm{N}_{\mathrm{A}} \\
\left(\mathrm{cm}^{-3}\right)\end{array}$ \\
\hline $\begin{array}{l}\text { dark } \\
\text { dot }\end{array}$ & 0.46 & 0.060 & $\begin{array}{c}3.75 \cdot 10^{17} \\
(*)\end{array}$ \\
\hline $\begin{array}{l}\text { bright } \\
\text { halo }\end{array}$ & 0.73 & 0.097 & $1.43 \cdot 10^{17}$ \\
\hline matrix & 0.71 & 0.065 & $3.20 \cdot 10^{17}$ \\
\hline
\end{tabular}

(*) See text.

Fig.2 - Dot-and-halo features at dislocations in a GaAs crystal. Dopant striations can also be seen. The bar is $50 \mu \mathrm{m}$.

The density of the net ionized shallow impurities, $N_{D}-N_{A}$, given in Table $I$, is evaluated by the well known formula ${ }^{2}$

$$
N_{D}-N_{A}=2 \varepsilon V_{0} /\left(q W^{2}\right)
$$

where $N_{D}$ and $N_{A}$ are the ionized donor and acceptor densities, respectively, $\varepsilon$ the dielectric constant, $V_{0}$ the built-in potential of the diode, and $q$ the electron charge. The increase of $W$ thus means that there is a depletion of the net ionized shallow impurities in the bright halo with respect to the surrounding matrix. Assuming that before depletion has occurred the doping level in the areas that now give EBIC bright contrast was the same as in the matrix, Table I shows that such areas have been depleted by an amount $N_{D}-N_{A}=1.77 \cdot 10^{17} \mathrm{~cm}^{-3}$. However, $N_{D}-N_{A}$ in the dark dot did not increase by the same amount. This has been suggested as being due to the fact that the dislocation might also have gettered deep impurities of both acceptor and donor type that can affect the value of $W$ at the dislocation impurity atmosphere. ${ }^{8}$ In such a case eq. (3) is no longer valid and a more complex relationship between $W$ and the densities of all the ionized impurities must be used.8 $N_{D}-N_{A}$ in the dark dot in Table I must be regarded as approximate and giving only a rough indication (increase/decrease) of the behaviour of the net density of all the electrically active impurities. It must be expected, however, that the atoms that left the bright halos have diffused to the dislocation rather than to the matrix because of the gettering action of the dislocation. 
The increase of $L$ in the bright halos, if any, was rather due to a depletion of deep impurities, i. e. reduced non-radiative recombination. The depletion of shallow impurities, as measured here, cannot account for the change of $L$. The measured value of $L$, in fact, is determined by the smallest one between $L_{R}$ and $L_{N R}$ as it is given by

$$
1 / L^{2}=\left(1 / L_{R}\right)^{2}+\left(1 / L_{N R}\right)^{2}=B n / D+\left(v_{t h} \Sigma_{i} \sigma_{i} N_{T i}\right) / D
$$

where $L_{R}$ and $L_{N R}$ are the radiative (band-to-band) and non-radiative diffusion lengths, respectively, $D$ the hole diffusion coefficient $\left(10 \mathrm{~cm}^{2} / \mathrm{sec}\right), B$ a constant equal to $1.7 \cdot 10^{-10}$ $\mathrm{cm}^{3} / \mathrm{sec},{ }^{9} v_{\text {th }}$ the thermal velocity of the carriers, $\sigma_{i}$ and $N_{T i}$ the capture cross section and density of the ith deep impurity, respectively, and $n$ the free electron concentration. In this sample $n=2.0 \cdot 10^{17} \mathrm{~cm}^{-3}$ and $L_{R}=5.6 \mu \mathrm{m}$ so that the low measured values of $L$ (see Table $\mathrm{I}$ ) would indicate that $L$ is mostly affected by non-radiative recombination at deep impurities.
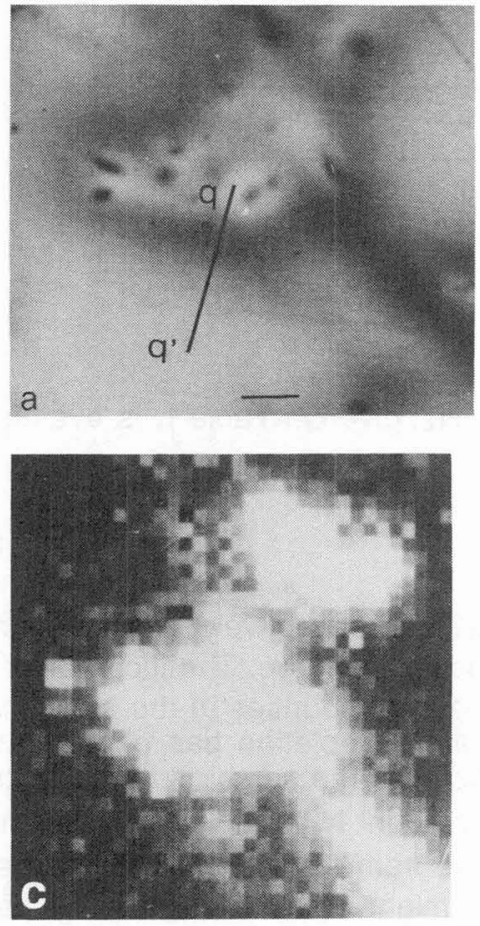

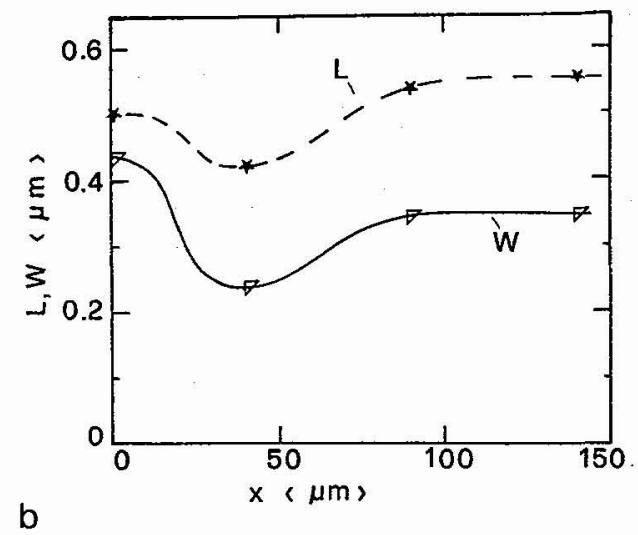

Fig. 3 - a) EBIC image of dot-and-halo features. b) $L$ and $W$ variation along line $q-q^{\prime}$ in $\left.a\right) ; x=0 \mu m$ corresponds to the maximum EBIC signal inside the bright halo and $x>\sim 100 \mu \mathrm{m}$ corresponds to the matrix. c) SDLTS (EL2 level) image of the same area as in a). A bright SDLTS signal means increased density of the selected deep trap. The bar is 50 $\mu \mathrm{m}$. (From Ref. [11], by courtesy of Elsevier Sci. Pu. B.V.).

Scanning Deep Level Transient Spectroscopy (SDLTS) ${ }^{10}$ and photoluminescence (PL) have been used to check the EBIC conclusions and full discussion of the results is given in Refs. $[11,12]$. Fig. 3 a) is an EBIC image of dislocations surrounded by bright halos in a GaAs sample Si-doped to $\sim 1.0 \cdot 10^{16} \mathrm{~cm}^{-3}$ and grown from an As-rich melt. Even in this sample $\mathrm{W}$ was greater (by $\sim 20 \%$ ) within the bright halos than in the matrix. This corresponds to a reduction of $\sim 30 \%$ of the net ionized shallow (donor) impurities in the bright halo with respect to the defect-free matrix. ${ }^{11}$ On the other hand, in this case $L$ decreased by $10 \%$ with respect to the matrix (Fig. 3 b). A depletion of deep impurities within the areas of negative EBIC 
contrast is, therefore, not expected. Also in this case, in fact, according to eq. (4) $L$ is mostly affected by non-radiative recombination processes at deep traps as its value ranged from 0.50 to $0.55 \mu \mathrm{m}$, being thus much lower than $L_{R}=24 \mu \mathrm{m}$.

SDLTS mappings confirmed such expectations as they did not show any appreciable change of the density of the most important deep levels. ${ }^{11}$ Fig $3 \mathrm{c}$ ) is an SDLTS image of the same area as in Fig. 3 a) showing, for instance, the distribution of the EL2 deep level ( $E_{C^{-}}$ $0.75 \mathrm{eV}$ ). It is evident that no depletion of EL2 has occurred within the areas corresponding to the EBIC bright halos and that the distribution of EL2 is pretty homogeneous over nearby areas exibiting dark EBIC contrast. The other traps detected by SDLTS, i.e. the hole traps HB5 and HB6, also exhibited the same behaviour as EL2 .11
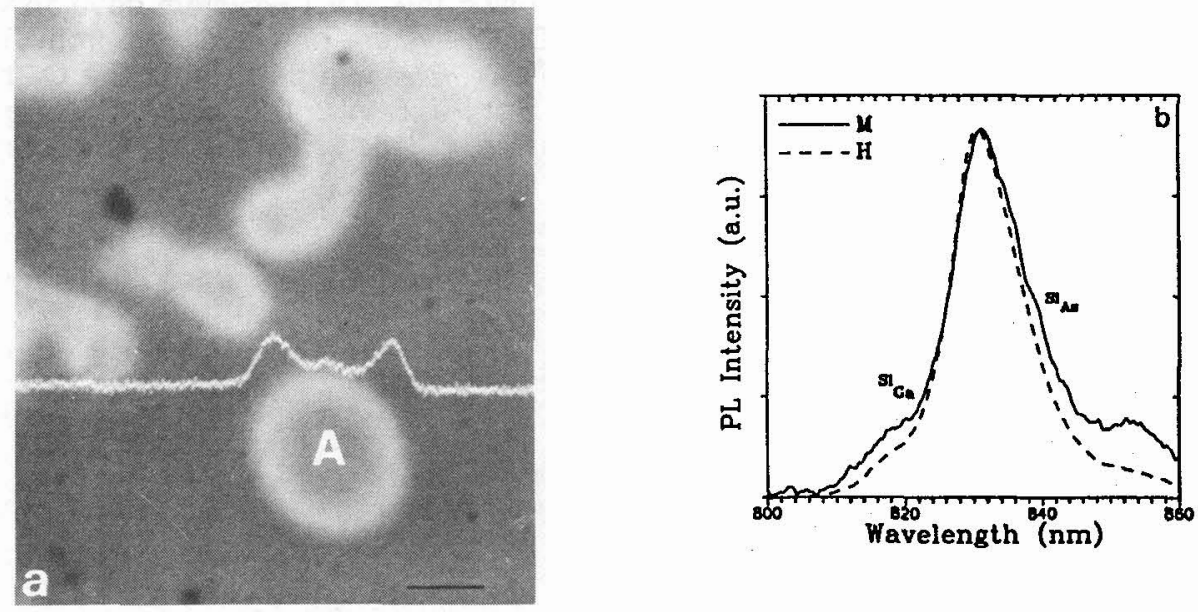

Fig. 4 - a) EBIC image of areas with negative EBIC contrast in a GaAs sample grown from a Ga-rich melt. The EBIC line scan was taken along the diameter of feature A. The bar is $50 \mu \mathrm{m}$. b) PL spectra taken from the bright halo $(\mathrm{H})$ of feature $A$ in the EBIC image in a) and from the nearby matrix (M). (From Ref. [12], by courtesy of Elsevier Sci. Pu. B.V.).

The decrease of the dopant density within the EBIC bright halos was also confirmed by PL investigations. ${ }^{12} \mathrm{Fig} .4 \mathrm{a}$ ) is the EBIC image from a GaAs sample Si-doped to $4.10^{17} \mathrm{~cm}^{-3}$ and grown from a Ga-rich melt. Several areas exhibiting bright contrast are in the field of view. The characteristics and origin of these defects have been discussed elsewhere. ${ }^{12}$ Again, the EBIC measurements showed that the dopant density is lower in the bright areas than in the surrounding areas, whilst $L$ is higher. In the brightest annular halo of feature $A$, for instance, $N_{D}-N_{A}$ was $6.6 \cdot 10^{15} \mathrm{~cm}^{-3}(\mathrm{~L}=2.7 \mu \mathrm{m})$ whereas in the matrix it was $3.7 \cdot 10^{17} \mathrm{~cm}^{-3}(\mathrm{~L}$ $=0.75 \mu \mathrm{m})$. Such great change in $N_{D}-N_{A}$ was also confirmed ${ }^{12}$ by the formation of very deep grooves at such defects when the specimen underwent DSL photoetching ${ }^{13}$ whose etching rate strongly depends on the dopant density. ${ }^{14}$ Fig. 4 b) shows the PL spectra (from Ref. [12]) taken from the annular EBIC bright halo $(H)$ of feature $A$ and from the nearby matrix (M) using a PL apparatus with high spatial resolution $(2 \mu \mathrm{m})$ working at $4.2^{\circ} \mathrm{K}$. The $\mathrm{PL}$ spectra clearly show that the level of $\mathrm{Si}$, in both the As and Ga sublattice, is higher in the matrix than in the halo. In samples similar to that of Fig. 1, PL also confirmed that in the impurity atmospheres at the dislocations there is an increase of the density of the dopant Si, mostly located in the Ga sublattice, ${ }^{15}$ as found by EBIC (Table I and Ref. [8]). 


\section{4. - Conclusions}

By localized quantitative measurements of $W$ and $L$ by energy-dependent $E B I C$ it has been found that the EBIC bright halos are mostly due to an increase of the SCR width $W$ rather than to an increase of L. PL confirmed the EBIC result that in such bright halos there is a depletion of shallow donor impurities ${ }^{12}$ whereas SDLTS supported the other EBIC result that the depletion of deep non-radiative impurities is sometimes negligible. ${ }^{11}$

\section{Acknowledgements}

The SDLTS image was taken by Dr. O. Breitenstein using the SDLTS-system developed at the IFE Halle, being the basis of the commercial SDLTS-system now available from Raith $\mathrm{GmbH}$ Dortmund (D). The PL spectra were taken by Dr. P.J. van der Wel at the Catholic University, Nijmegen (NL). Permission from Elsevier Sci. Pu. B.V. to reproduce here Figs. 3 and 4 is greatly acknowledged. Thanks are due to Mr. P. Allegri for the skilful technical assistance in the preparation of the diodes.

\section{References}

11/ Holt,D B, in SEM Microcharacterization of Semiconductors, Holt,D B, and Joy,D C, Eds., Academic Press, London, 1989, Ch. 6

12/ Frigeri,C, Inst. Phys. Conf. Ser. 87 (1987) 745

13/ Seifert,W, and Kittler,M, Phys. Stat. Sol. (a) 99 (1987) K11

14/ Blumtritt,H, Kittler,M, and Seifert,W, Inst. Phys. Conf. Ser. 104 (1989) 233

15/ Yacobi,B G, and Holt,D B, Cathodoluminescence Microscopy of Inorganic Solids, Plenum Press, New York, 1990 , ch. 6

16/ Wu,C J, and Wittry,D B, J. Appl. Phys. 49 (1978) 2827

I7/ Frigeri, $\mathrm{C}$, to be published

18/ Frigeri,C, and Weyher,J L, J. Appl. Phys. 65 (1989) 4646

19/ Ryan,R D, and Eberhart,J S, Solid St.-Electron 15 (1972) 862

/10/ Heydenreich,J, and Breitenstein, O, J. Microsc. 141 (1985) 273

111/Frigeri, C, and Breitenstein, $\mathrm{O}$, in Defect Control in Semiconductors, Sumino,K, Ed., Elsevier, Amsterdam, 1990,685

112/ Weyher,J L, Frigeri,C, and van der Wel,P J, J. Crystal Growth 103 (1990) 46

113/ Weyher,J L, and van de Ven,J, J. Crystal Growth 78 (1986) 191

114/ Frigeri,C, and Weyher,J L, J. Crystal Growth 103 (1990) 268

115/ Visser,E P, van der Wel,P J, Weyher,J L, and Giling, L J, J. Appl. Phys. 68 (1990) 4242. 